\title{
Quasiparticles near quantum phase transition in heavy fermion metals
}

\author{
V.R. Shaginyan *, \\ Petersburg Nuclear Physics Institute, RAS, Gatchina 188300, Russia
}

\begin{abstract}
We have shown that the Landau paradigm based upon both the quasiparticle concept and the notion of the order parameter can be used to explain the anomalous behavior of heavy fermion metals. Exploiting this paradigm and the fermion condensation quantum phase transition (FCQPT) we show that this anomalous behavior is universal and can be used to capture the essential aspects of recent experiments on the heavy-fermion metals at low temperatures. Behind FCQPT a tunneling conductivity between a heavy fermion metal and a simple metallic point can be noticeably dissymmetrical with respect to the change of voltage bias. We show that at $T=0$ and beyond FCQPT the Hall coefficient undergoes a jump upon magnetic-field tuning HF metals.
\end{abstract}

Key words: quantum phase transitions, heavy fermions, universal behavior, dissymmetrical tunneling, Hall coefficient PACS: 71.10.Hf; 71.27.+a; $75.30 \mathrm{Cr}$

Experiments on heavy-fermion (HF) metals explore mainly their thermodynamic properties which proved to be quite different from that of ordinary metals described by the Landau Fermi liquid (LFL) theory. In the LFL theory, considered as the main instrument when investigating quantum many electron physics, the effective mass $M^{*}$ of quasiparticle excitations controlling the density of states determines the thermodynamic properties of electronic systems. It is possible to explain many of the observed properties of the HF metals on the basis of FCQPT which is in harmony with the Landau paradigm resting upon both the quasiparticle concept and the notion of the order parameter. In contrast to the conventional Landau quasiparticles, these are characterized by the effective mass which strongly depends on temperature $T$, applied magnetic field $B$ and the number density $x$ of the heavy electron liquid of HF metal.

To study the universal behavior of the HF metals at low temperatures we use a heavy electron liquid

\footnotetext{
* Corresponding author

Email address: vrshag@thd.pnpi.spb.ru (V.R. Shaginyan).
}

model in order to get rid of the specific peculiarities of HF metals. Since we consider processes related to the power-low divergency of the effective mass, this divergency is determined by small momenta transferred as compared to momenta of the order of the reciprocal lattice, and the contribution coming from the lattice can be ignored. We start with the well-known Landau equation determining $M^{*}(T, B)$

$$
\frac{1}{M^{*}(T, B)}=\frac{1}{M}+\int \mathbf{Y} F\left(\mathbf{p}_{\mathbf{F}}, \mathbf{p}_{1}\right) \frac{\partial n\left(\mathbf{p}_{1}\right)}{\partial p_{1}} \frac{d \mathbf{p}_{1}}{(2 \pi)^{3}}
$$

For brevity, we omit spin variables. Here $\mathbf{Y}=$ $\left(\mathbf{p}_{F} \mathbf{p}_{\mathbf{1}}\right) / p_{F}^{3}, F\left(\mathbf{p}_{\mathbf{F}}, \mathbf{p}_{1}\right)$ is the Landau amplitude depending on the momenta $p, p_{F}$ is the Fermi momentum, $M$ is the bare mass of an electron, and $n\left(\mathbf{p}_{1}, T, B\right)$ is the quasiparticle distribution function. Applying Eq. (1) at $T=0$ and $B=0$, we obtain the standard result $M^{*}(x)=M /\left(1-N_{0} F^{1}\left(p_{F}, p_{F}\right) / 3\right)$. Here $N_{0}$ is the density of states of the free Fermi gas and $F^{1}\left(p_{F}, p_{F}\right)$ is the $p$-wave component of the Landau interaction. Since in the LFL theory $x=p_{F}^{3} / 3 \pi^{2}$, the Landau amplitude can be written as $F^{1}\left(p_{F}, p_{F}\right)=$ $F^{1}(x)$. Assume that at some critical point $x_{F C}$ the 
denominator $\left(1-N_{0} F^{1}(x) / 3\right)$ tends to zero, and one obtains that $M^{*}(x)$ behaves as $M^{*}(x) / M \propto 1 / r$. Here $r=\left(x-x_{F C}\right)$ is the "distance" from the QCP of FCQPT taking place at $x_{F C}$.

At $r \rightarrow 0$ and $T<T^{*}\left(B-B_{c 0}\right) \propto\left(B-B_{c 0}\right)$, the system has the LFL behavior and a qualitative analysis of Eq. (1) shows that $M^{*}(B) \propto\left(B-B_{c 0}\right)^{-2 / 3}$. Here, $B_{c 0}$ is the critical magnetic field which drives both a $\mathrm{HF}$ metal to its magnetic field tuned QCP and the corresponding Néel temperature $T_{N} \rightarrow 0$ [1]. At elevated temperatures the system demonstrates two types of the non-Fermi liquid behavior (NFL): at $T \sim T^{*}(B)$ $M^{*}(T) \propto T^{-2 / 3}$, and $T>T^{*}(B) M^{*}(T) \propto 1 / \sqrt{T}$. These mentioned regimes can be observed in measurements of the resistivity, $\rho(T)=\rho_{0}+\Delta \rho$. Here, $\rho_{0}$ is the residual resistivity, $\Delta \rho=A(B) T^{2}$, and $A(B) \propto$ $\left(M^{*}\right)^{2}$. The first LFL regime is represented by $\Delta \rho_{1} \propto$ $T^{2} /\left(B-B_{c 0}\right)^{4 / 3} \propto T^{2}$; the second NFL one is characterized by $\Delta \rho_{2} \propto T^{2} /\left(T^{2 / 3}\right)^{2} \propto T^{2 / 3} ;$ and the third NFL one is represented by $\Delta \rho_{3} \propto T^{2} /(\sqrt{T})^{2} \propto T$. All these regimes were observed in recent measurements on the HF metals, see e.g. [2]. Considering the ratio $\Delta \rho_{2} / \Delta \rho_{1} \propto\left(\left(B-B_{c 0}\right) / T\right)^{4 / 3}$, we conclude that the ratio is a function of only the variable $\left(B-B_{c 0}\right) / T$ representing the scaling behavior. This result is in excellent agreement with experimental facts [2].

Beyond the critical point $x_{F C}$ the distance $r$ becomes negative making the effective mass negative. To escape the possibility of being in unstable and meaningless states with the negative effective mass, the system is to undergo FCQPT quantum phase transition at the critical point $x=x_{F C}$ [1]. At $x<x_{F C}$ the quasiparticle distribution is determined by the standard equation to search the minimum of a functional $\delta E[n(\mathbf{p})] / \delta n(\mathbf{p}, T=0)=\varepsilon(\mathbf{p})=\mu ; p_{i} \leq p \leq p_{f}$. This equation determines the quasiparticle distribution function $n_{0}(\mathbf{p})$ which delivers the minimum value to the ground state energy $E$. $n_{0}(\mathbf{p})$ does not coincide with the step function in the region $\left(p_{f}-p_{i}\right)$, so that $0<n_{0}(\mathbf{p})<1$, while $p_{i}<p_{F}<p_{f}$. This special behavior of $n_{0}(\mathbf{p})$ determines the behavior of system at $T<T_{f}$, with $T_{f}$ being the temperature at which he influence FCQPT vanishes. At $T=0$, the relevant order parameter is the superconducting-like, $\kappa(\mathbf{p})=$ $\sqrt{n_{0}(\mathbf{p})\left(1-n_{0}(\mathbf{p})\right)}$, with the entropy $S=0[1]$. At $0<$ $T<T_{f}$, the entropy can be approximated as $S(T) \simeq$ $S_{0}+a \sqrt{T / T_{f}}, a$ is a constant. This temperature independent term $S_{0}$ determines the specific NFL behavior of the system. For example, the thermal expansion coefficient $\alpha(T)$ determined by the contribution coming from $S_{0}$ becomes constant at $T \rightarrow 0$ while the specific heat $C \sim a \sqrt{T / T_{f}}$. As a result, the Grüneisen ratio $\Gamma$ diverges $\Gamma=\alpha / C \propto 1 / \sqrt{T}$. Then, at $T<T_{f}$, a tunneling conductivity between a heavy fermion metal and a simple metallic point can be noticeably dissymmet- rical with respect to the change of voltage bias [3]. At $T<T^{*}\left(B-B_{c 0}\right) \propto \sqrt{B-B_{c 0}}$, the application of magnetic field $B$ restores the LFL behavior, the effective mass becomes $M^{*}(B) \propto 1 / \sqrt{B-B_{c 0}}$, and the coefficient $A \propto 1 /\left(B-B_{c 0}\right)[1]$. At $T=0$, the application of the critical magnetic field $B_{c 0}$ suppressing the antiferromagnetic state $(\mathrm{AF})$ (with the Fermi momentum $\left.p_{A F} \simeq p_{F}\right)$ restores the LFL with the Fermi momentum $p_{f}>p_{F}$. Both AF and LFL have the same ground state energy being degenerated at $B=B_{c 0}$. Thus, at $T=0$ and $B=B_{c 0}$, the infinitesimal change in the magnetic field $B$ leads to the finite jump in the Fermi momentum. In response the Hall coefficient $R_{H} \propto 1 / x$ undergoes the corresponding sudden jump.

As an illustration of the above consider the $T-B$ phase digram for $\mathrm{YbRh}_{2} \mathrm{Si}_{2}[4]$ (Fig. 1). Taking into account that the behavior of $\mathrm{YbRh}_{2} \mathrm{Si}_{2}$ strongly resembles the behavior of $\mathrm{YbRh}_{2}\left(\mathrm{Si}_{0.95} \mathrm{Ge}_{0.05}\right)_{2}$ [5], we can conclude that both the above described $T-B$ diagram and the behavior of the Hall coefficient $R_{H}$ are in good agreement with experimental facts $[4,5]$.

This work was supported by Russian Foundation for Basic Research, Grant No 05-02-16085.

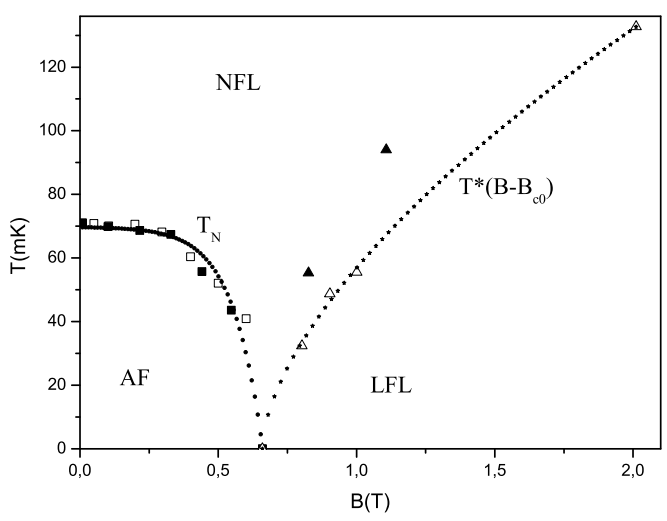

Fig. 1. Line separating AF and the NFL state is a guide to the eye, line separating the NFL and the LFL phases $T^{*}\left(B-B_{c 0}\right) \propto \sqrt{B-B_{c 0}}[1]$.

References

[1] V.R. Shaginyan, JETP Lett. 79 (2004) 286; V.R. Shaginyan, A.Z. Msezane, M.Ya. Amusia, Phys. Lett. A 338 (2005) 393.

[2] J. Paglione et al., cond-mat/0405157

[3] V.R. Shaginyan, JETP Lett. 81 (2005) 222.

[4] P. Gegenwart et al., Phys. Rev. Lett. 89 (2002) 056402. 
[5] R. Küchler et al., Phys. Rev. Lett. 91 (2003) 066405; J. Custers et al., Nature 424 (2003) 524; S Paschen et al., Nature 432 (2004) 881. 